\title{
Processamento de Áudio em Microcontroladores Simples
}

Title: Audio Processing With Simple Microcontrollers

Adilson Cavalcanti ${ }^{1}$ (D) http://orcid.org/0000-0002-0800-1716

Sérgio Campello Oliveira ${ }^{1}$ (1) http://orcid.org/0000-0003-1058-1139

${ }^{1}$ Escola Politécnica de Pernambuco, Universidade de Pernambuco, Recife, Brasil,

E-mail do autor principal: Adilson Cavalcanti adilsonchacon@gmail.com

\section{Resumo}

Este artigo descreve o desenvolvimento de dispositivo eletrônico capaz de realizar processamento de áudio em hardware utilizando microcontroladores de baixo custo. São descritos hardwares para a captação e amplificação de áudio. Demonstra como o microcontrolador deve receber os sinais de áudio, como deve processa-los e como deve fornecer os dados de saída. O sinal processado foi enviado através da saída PWM para o amplificador e ao alto falante. Para comprovar o adequado processamento e a inteligibilidade dos áudios processados, testes com voluntários foram realizados. Os testes demonstraram $100 \%$ de inteligibilidade, comprovando a eficácia do método utilizado.

Palavras-Chave: Processamento; áudio; amplificador; microcontrolador; pwm.

\begin{abstract}
This paper describes the development of an electronic devices that process audio by hardware using low costs microcontrollers. Describe hardware for audio capitation and amplification. Shows hardware with audio capture and amplification. Demonstrates how a microcontroller should receive the sounds signals, how to process them and how to supply the output data. The processed signal was sent through the PWM output to the amplifier and the speaker. To prove the correct processing and intelligibility of the audio, tests with volunteers were performed. The tests showed $100 \%$ of intelligibility, proving the efficacy of the method used.
\end{abstract}

Key-words: Processing; audio; amplifier; microcontroller; pwm. 


\section{Introdução}

Este artigo descreve como processar áudio em um sistema embarcado. São descritas a captação da onda sonora de um ambiente, sua transformação em sinais elétricos, conversão analógico-digital com uso de um microcontrolador simples, como o microcontrolador processa os dados digitais e como consegue entregar o resultado ao alto falante com o máximo de qualidade.

Alguns desafios apareceram durante a implementação. Dentre eles podemos destacar o fato que o microcontrolador utilizado não trabalha com números negativos e uma onda de som contem valores positivos e negativos. Logo o amplificador para o microfone deve elevar toda a amplitude da onda sonora para valores positivos.

Mesmo com a limitação de processamento do microcontrolador, teve-se o cuidado em ter o mínimo de perda de qualidade das ondas sonoras ao remontala após o processamento interno do microcontrolador.

Ao terminar a leitura desse artigo os(as) leitores(as) terão o conhecimento básico para implementar qualquer dispositivo eletrônico de áudio, como gravadores de som e rádios comunicadores, utilizando microcontroladores.

\section{Revisão Bibliográfica}

\subsection{PWM}

PWM (Pulse Width Modulation), ou modulação por largura de pulso, é uma onda quadrada de amplitude máxima constante e frequência variável, cuja tensão média substitui uma tensão contínua. A tensão média varia em função do tempo em que a onda fica em nível alto (VCC) e do tempo em que a onda fica em nível baixo (OV). A relação entre o tempo em que a onda fica em nível alto e o período total é chamado de Duty Cycle. Por exemplo, uma tensão de $5 \mathrm{~V}$ e um Duty Cycle de $50 \%$ tem o mesmo efeito que uma função contínua de 2,5V [1], como mostra a Figura 1.

Para o PWM representar uma frequência especifica é necessário um número limitado de combinações de Duty Cycle que chamamos de resolução. Por exemplo, se a resolução máxima do microcontrolador for de 10 bits, são necessários 1024 passos para representar o Duty Cycle. Lembrando que o Duty Cycle é dado em porcentagem (0\% a 100\%), assim para uma resolução de 10 bits teremos o valor 0 como $0 \%$ e 1023 para $100 \%$.

\subsection{Desenvolvimentos Semelhantes}

R. Copîndean, R. Holonec e F Drăgan [2] desenvolveram um sistema de captura e processamento de áudio.

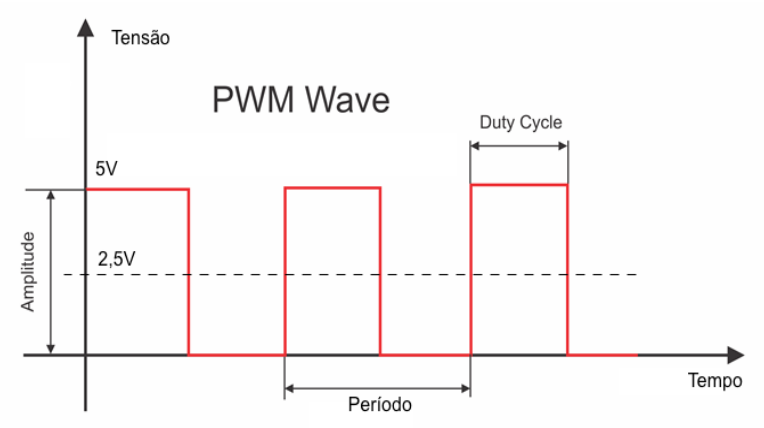

Figura 1: Exemplo de PWM. Fonte: Internet.

Por microcontrolador. O sistema capta o áudio através de um microfone, em seguida envia o sinal para um pré-amplificador e depois entrega os dados ao microcontrolador pela entrada analógica. O sistema usa, ainda, um segundo dispositivo de entrada para fazer a mixagem com outro dispositivo através de um circuito somador. O microcontrolador utilizado em [2] foi um ATmega 328 [3], que possui processador de 8 bits e clock de $20 \mathrm{MHz}$.

S. Vinod [4] desenvolveu um sistema de gravador som. As frequências de áudio capturadas pelo microfone, passadas para a entrada analógica do microcontrolador e salva em um cartão de memória. E depois foi possível ouvir o áudio gravado. Para esse projeto foi usado um microcontrolador PIC 16F877A [5] que possui processador de 8 bits e clock máximo de $20 \mathrm{MHz}$.

L. Bengtsson [6] desenvolveu um circuito para melhorar a qualidade do som tanto para entrada como para saída eliminando ruídos. Para esse projeto foi usado um microcontrolador PIC 18F458 [7] que possui processador de 8 bits e clock máximo de $40 \mathrm{MHz}$.

O microcontrolador utilizado neste trabalho foi o PIC $18 \mathrm{~F} 4550$ [8], que possui processador de 8 bits e clock máximo de $48 \mathrm{MHz}$. 


\subsection{Conversão Analógico-Digital}

Os conversores Analógicos-Digitais são capazes de converter tensões analógica em valores digitais [9]. Para o microcontrolador PIC $18 \mathrm{~F} 4550$ a conversão Analógico-Digital tem resolução de 10 bits e o ciclo é formado pelo tempo de aquisição, o tempo de conversão de cada bit e também o tempo de espera entre um ciclo e outro. O tempo de conversão de cada bit é chamado de TAD que é definido pelo período do sinal de clock do conversor. Há dois TADs extras, um antes da conversão dos 10 bits e um outro no final, então podemos definir o tempo total de conversão de acordo com a equação (1), vejamos:

Ciclo de Conversão $=$ Tempo de Conversão $+12 x$ TAD + Tempo de Espera

O Tempo de Conversão, segundo o datasheet, é de 2,4 us. O TAD é configurável e configuramos para 1 us. O Tempo de espera é o dobro do TAD, logo temos:

Ciclo de Conversão $=2,4 u s+12 \times 1$ us +2 us

Ciclo de Conversão $=16,4$ us

Isso significa que a cada conversão haverá uma perda de 16,4us de áudio.

\section{Desenvolvimento}

Para implementação do sistema é necessário um microfone, um circuito amplificador de sinal, um microcontrolador com hardware potente o suficiente para realizar a tarefa que a aplicação precisa, um circuito amplificador de potência e um alto falante.

O microfone vai captar a onda de som transformando-a em sinais elétricos e vai entrega-los para o circuito amplificador de sinais. O circuito amplificador de sinais vai fazer um aumento da amplitude em toda onda de som para que ela possa ser lida pelo microcontrolador que receberá os dados analógicos, vai remontar a onda sonora através do PWM e vai encaminhar os dados ao circuito amplificador de potência para que ganhe amplitude o suficiente para ser reproduzido em um alto falante. Como mostra a Figura 2.

Todos os elementos usados no circuito foram escolhidos pela facilidade de compra no mercado local.

\subsection{Microfone e Amplificador de Sinais}

Um microfone de eletreto foi usado como entrada para o amplificador de sinais [10]. O Amplificador de sinais deve elevar toda a amplitude da onda de som para valores positivos, pois o microcontrolador em uso não aceita valores negativos.

Veja o circuito amplificador simples na Figura 3.

Para testar o sistema foi utilizado tom de $17000 \mathrm{~Hz}$ em sua entrada. Com auxílio de um osciloscópio foi possível verificar a onda original na figura 4 e a onda amplificada na figura 5 .

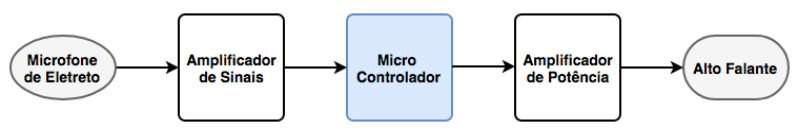

Figura 2: Fluxo de dados Completo. Fonte: Software http://draw.io.

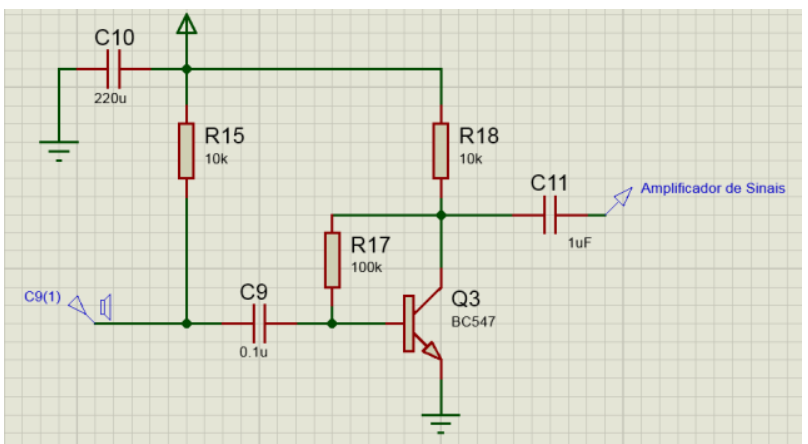

Figura 3: Esquema elétrico do amplificador de sinais. Fonte: Software Proteus.

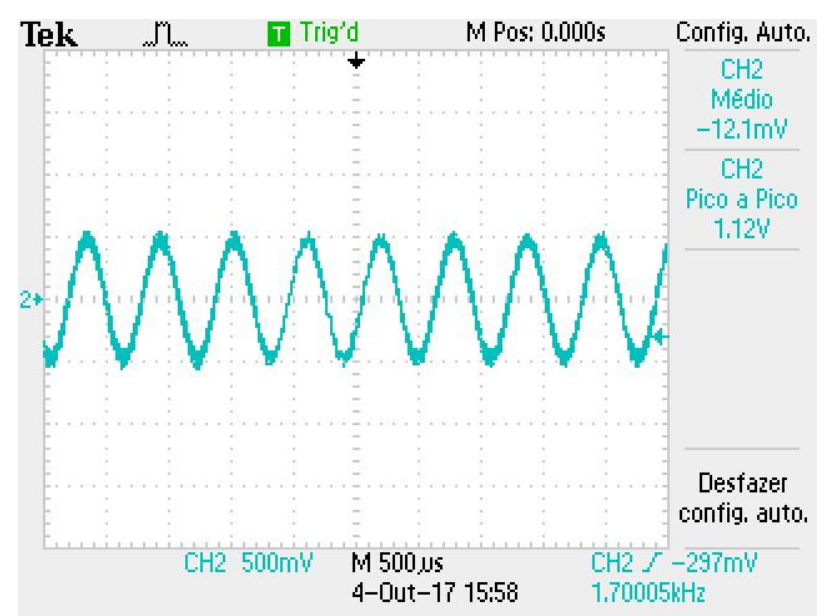

Figura 4: Onda de som original. Fonte: Osciloscópio do Laboratório de Engenharia Elétrica da Poli-UPE 


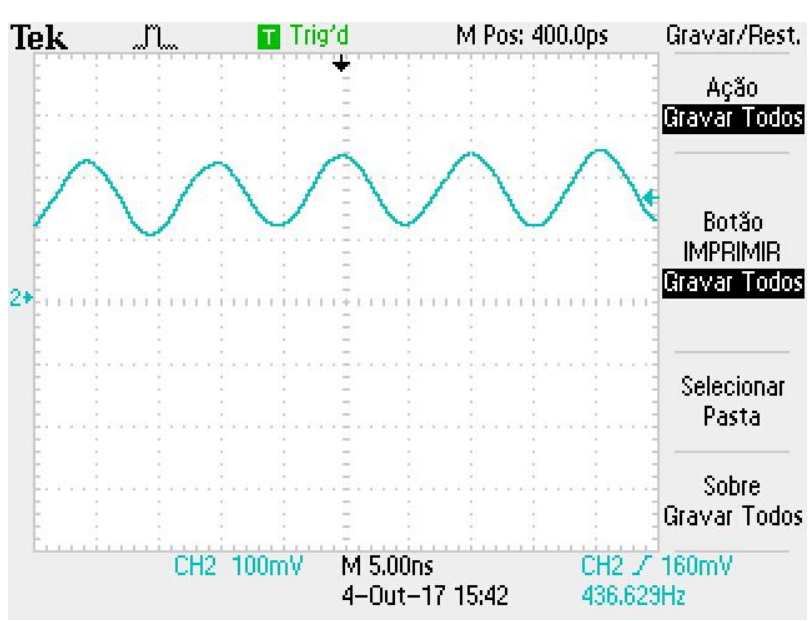

Figura 5: Onda de som amplificada. Fonte: Osciloscópio do Laboratório de Engenharia Elétrica da Poli-UPE

\subsection{Microcontrolador}

Utilizamos o PIC 18F4550. Seu clock externo é composto de um cristal oscilador de $4 \mathrm{MHz}$ e de dois capacitores de $22 \mathrm{pF}$.

O áudio amplificado entra pela entrada analógica (ANO) sendo convertido em sinal digital.

Para a saída é preciso remontar a onda sonora através dos dados digitais. Para isso usamos o recurso do PWM do microcontrolador. Na Figura 6 temos um exemplo que compara uma onda de som ao PWM.

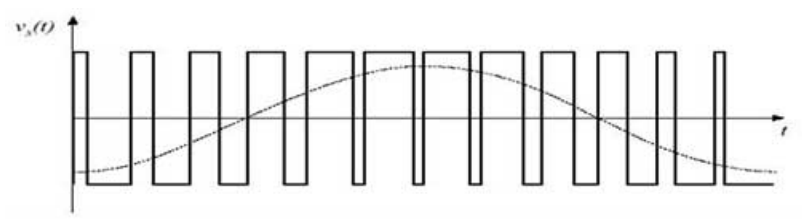

Figura 6: Comparação entre uma onda de som e ondas quadradas geradas pelo PWM. Fonte: Internet.

Para configuração do PWM utilizamos o TIMER2. Para isso devemos configurar o registrador PR2. O TIMER2 irá contar pulsos de clock do microcontrolador e quando for igual ao valor do PR2 então produzirá uma onda quadrada que ficará ativa de acordo com o tempo do designado pelo Duty Cycle. O TIMER2 novamente vai contar os pulsos de clock e quando for igual ao Duty Cycle o sinal voltará para zero. Dentre vários valores testados para o PR2, o valor 80 foi 0 que proporcionou melhor qualidade de som.

Com a configuração do PR2, podemos calcular o valor máximo do Duty Cycle aceito pelo PWM através da equação (3).

$$
\text { Duty Cycle }_{\text {MAX }}=4 *(P R 2+1)
$$

Logo o Duty Cycle máximo é 324, e o mínimo igual
a zero.

Na figura 7 é possível ver a saída do PWM, novamente com auxílio de um osciloscópio.

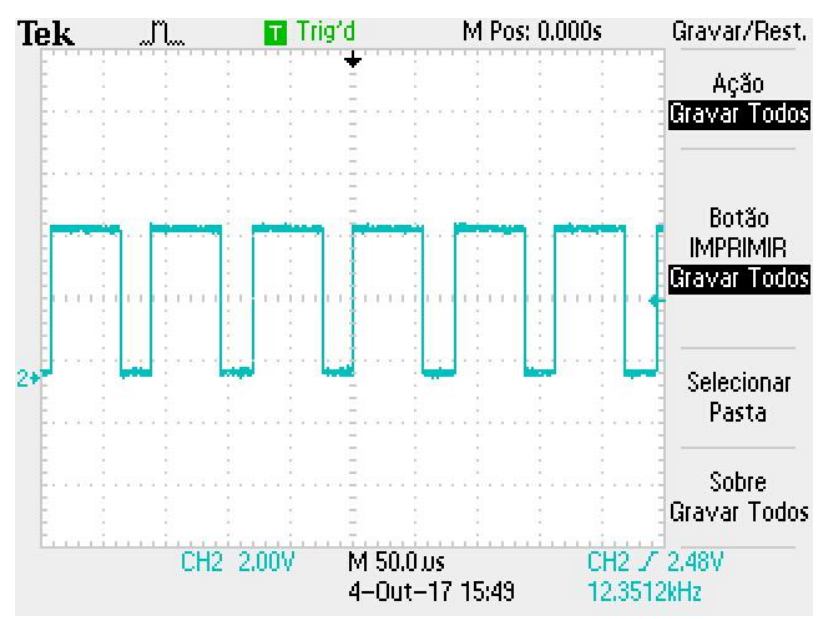

Figura 7: Onda de som gerada pelo PWM do microcontrolador. Fonte: Osciloscópio do Laboratório de Engenharia Elétrica da Poli-UPE.

\subsection{Amplificador de Potência e Alto Falante}

Quando sai do microcontrolador o pulso elétrico é enviado ao amplificador de potência para ganhar amplitude o suficiente para que o alto falante consiga reproduzir o som [11]. Confira o esquema elétrico na Figura 8.

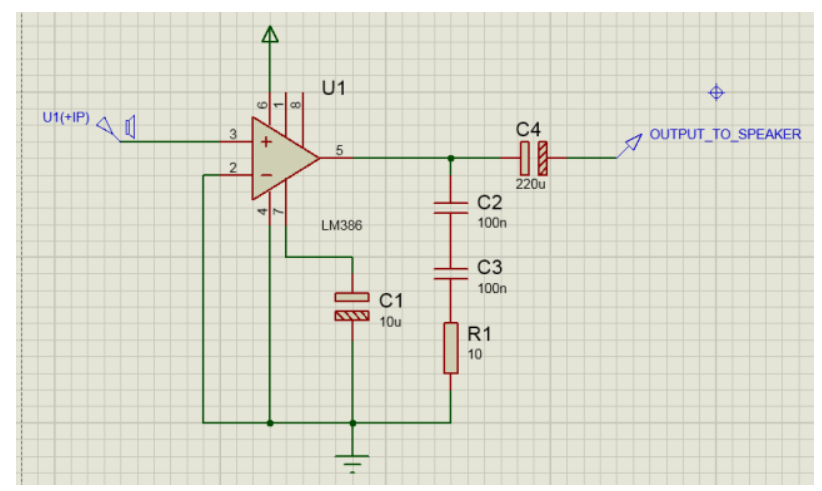

Figura 8: Esquema elétrico do amplificador de potência. Fonte: Software Proteus. 
O LM386 [12] é um amplificador de baixo consumo de tensão e de ganho interno de 20. Com o uso dos capacitores C1, C2 e C3, juntamente com o resistor R1, faz com que o ganho salte para 200.

\subsection{Montagem Completa}

Figuras para funcionamento correto dos dois amplificadores é necessária uma tensão de $9 \mathrm{~V}$. Porém o microcontrolador necessita uma tensão de 5V. Então decidimos que a alimentação central do sistema será de 9V. Um regulador de tensão LM7805 [13] será usado para reduzir a tensão para $5 \mathrm{~V}$ e alimentar o microcontrolador corretamente. Para o clock externo do microcontrolador será utilizado um cristal oscilador de $4 \mathrm{MHz}$.

Em posse dos componentes demonstrados, precisamos apenas conecta-los. Veja na Figura 9 que a alimentação do circuito está em 9V, ligada diretamente nos dois amplificadores e, indiretamente, no microcontrolador através do regulador de tensão. A saída do amplificador de sinais está conectada na entrada analógica do microcontrolador o qual sua porta do PWM serve de entrada para o amplificador de potência.

Na Figura 10 há uma montagem do sistema em protoboard.

\section{Testes}

Para testes contamos com cinco pessoas de língua nativa "Português do Brasil" de diferentes idades e profissões. Gravamos um áudio em um celular com o seguinte conteúdo: "Ontem choveu pela manhã, mas hoje está fazendo sol". Para cada pessoa, colocamos junto ao microfone do sistema embarcado a saída de áudio do celular em volume inaudível, executamos o áudio do celular, que foi captado pelo microfone, e pedimos para a pessoa ouvir, dizer se entendeu ou não e repetir a frase. Vejamos o resultado na Tabela 1.

\begin{tabular}{c|r|l|c}
\hline Nome & Idade & \multicolumn{1}{|c|}{ Profissão } & $\begin{array}{c}\text { Entendeu } \\
\text { e Repetiu }\end{array}$ \\
\hline Silvia & 37 & $\begin{array}{l}\text { Analista de } \\
\text { Sistemas }\end{array}$ & Sim \\
\hline Letícia & 7 & Estudante & Sim \\
\hline Cecília & 73 & Aposentada & Sim \\
\hline Tiago & 30 & $\begin{array}{l}\text { Engenheiro De } \\
\text { Segurança }\end{array}$ & Sim
\end{tabular}

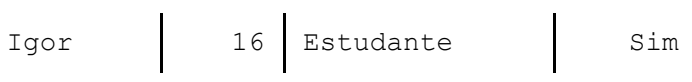

Tabela 1: Resultados dos testes. Fonte: Autor

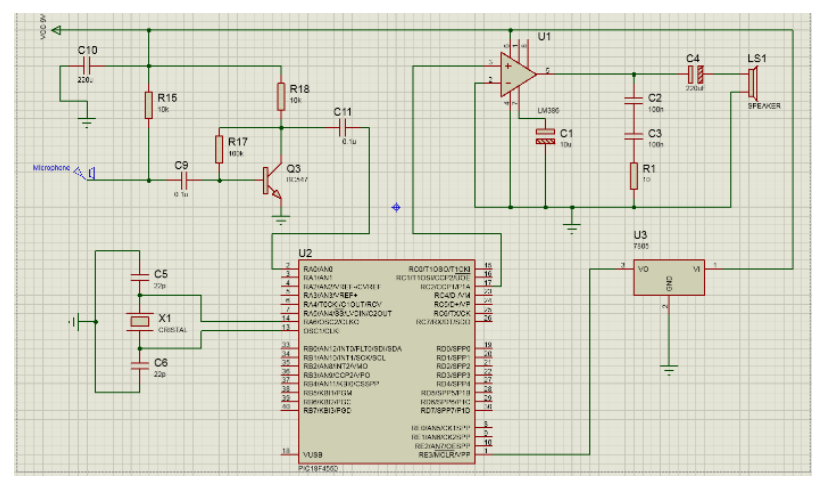

Figura 9: Esquema Elétrico Completo. Fonte: Software Proteus

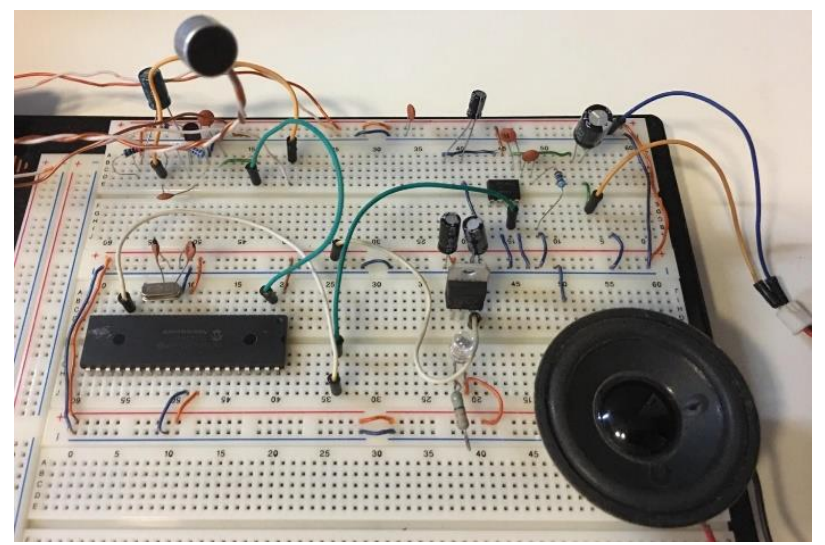

Figura 10: Foto da montagem na protoboard. Fonte: Autor

\section{Conclusões}

De acordo com os resultados dos testes, podemos afirmar que o sistema desenvolvido em geral funcionou com sucesso, com elementos de baixo custo e fácil de ser encontrados em mercado locais.

Então podemos afirmar, também, que é possível desenvolver outro sistema a partir do modelo apresentado. Como, por exemplo, um de gravador de som; com o microfone, o amplificador de sinais, o microcontrolador e com de um leitor de cartão de memória. Já com o Amplificador de Potência e o alto falante é possível desenvolver caixas acústicas de som. $E$, juntando tudo isso, é possível desenvolver um sistema completo de som, inclusive o seu próprio produto. 
Indo além, é possível desenvolver rádios comunicadores sem fio, e o alcance fica por conta do hardware usado para transmissão, inclusive pode-se usar módulos GSM para comunicação via celular.

E, por fim, é sempre interessante usar criptografia em dispositivos de comunicações para que a mensagem transmitida não seja entendida por pessoas indesejadas. Para isso recomendamos outros microcontroladores com poder computacional maior que o utilizado neste trabalho.

\section{Referências}

[1] Carlos Eduardo Sandrini Luz. Curso Linguagem $C$ para Microcontroladores PIC. Módulo PWM. 16(1):257.

[2] R Copîndean, R Holonec, F Drăgan. Audio Mixer Ordered Microcontroller. Acta Electrotehnica, Volume 56, Number 5, 221-224, 2015.

[3] Atmel Corporation 1600 Technology, ATmega 328/P. Datasheet, ATmel 8-BIT Microcontroller With 4/8/16/32 KBytes In-System Programmable Flash. Disponível em http://www.atmel.com/images/Atmel8271-8-bit-AVR-Microcontroller-ATmega48A-48PA-

88A-88PA-168A-168PA-328-

328P_datasheet_Complete.pdf. Acesso em: 12 de Junho de 2017.

[4] S. Vindur, An attempt to access a memory card (MMC) using a PIC with limited RAM (PIC16F877A). Disponível em: http://blog.vinu.co.in/2011/07/attempt-to-accessmemory-card-mmc-using.html. Acesso em 12 Junho de 2017.

[5] Microchip Technology Inc, Microchip PIC16F87XA Data Sheet, 28/40-Pin HighPerformance, Enhanced Flash Microcontrollers. Disponível em: http://ww1.microchip.com/downloads/en/DeviceDoc/ 39582b.pdf. Acesso em 12 de Junho 2017.

[6] L. E. Bengtsson. Interpolation Of Microcontroller ADC By Sself-Induced Dithering. International Journal On Smart Sensing And Intelligent Systems. Volume 6, Number 4, 1366-1382, 2013.
[7] Microchip Technology Inc, Microchip PIC18FXX8 Data Sheet, 28/40-Pin High-Performance, Enhanced Flash Microcontrollers with CAN Module. Disponível em: http://ww1.microchip.com/downloads/en/DeviceDoc/ 41159d.pdf. Acesso em 12 de Junho 2017.

[8] Microchip Technology Inc, Microchip PIC $18 \mathrm{~F}$ 2455/2550/4455/4550 Data Sheet, 28/40/44-Pin, High-Performance, Enhanced Flash, USB Microcontrollers with nano Watt Technology. Disponível em: http://ww1.microchip.com/downloads/en/DeviceDoc/ 39632e.pdf. Acesso em 12 de Junho de 2017.

[9] Carlos Eduardo Sandrini Luz. Curso Linguagem $C$ para Microcontroladores PIC. Módulo PWM. 10(1):155.

[10] Malvino, Albert. Eletrônica Volume I. Mac Graw Hill, Porto Alegre, 8a Edição. Amplificadores CC, BC e de múltiplos estágios, páginas 326-355. 2016.

[11] Malvino, Albert. Eletrônica Volume I. Mac Graw Hill, Porto Alegre, 8a Edição. Amplificadores de Potência, páginas 366-401

[12] Unisonic Technologies CO, LM386 Low Voltage Audio Power Amplifier

http://www.utcic.com/uploadfile/2011/0922/20110922090707869.p df . Acesso em 26 de Setembro de 2017.

[13] Fairchild Semiconductors, LM78XX/LM78XXA 3-Terminal 1 A Positive Voltage Regulator. Disponvível em: https://www.fairchildsemi.com/datasheets/LM/LM78 05.pdf. Acesso em 12 de Junho de 2017. 